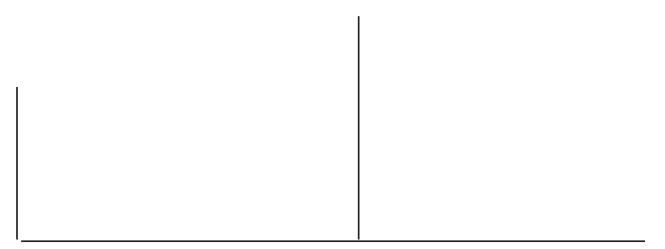

Rev. Latinoam. Psicopat. Fund., VII, 4, 112-134

\title{
Hontes sans issues... et issues de la honte: à propos d'un cas d'inceste
}

\author{
Delphine Scotto di Vettimo \\ Mário Eduardo Costa Pereira
}

\begin{abstract}
Le traitement psychothérapeutique des enfants ou des adultes victimes de traumatismes sexuels fait apparaître un sentiment de honte caractéristique. La première hypothèse ici envisagée prend en compte les deux instances psychiques de la personnalité que sont l'Idéal du Moi et le Moi-Idéal, dont les éléments déterminants sont : d'une part le rapport de l'Idéal du Moi avec l'expérience d'une atteinte sexuelle et de la blessure narcissique irréversible qu'elle provoque, d'autre part le Moi-Idéal en rapport avec la déchéance ressentie dans la honte consécutive au traumatisme sexuel, honte de n'être que la chose, l'objet, le déchet de l'agresseur. La deuxième hypothèse postule que dans le dispositif psychothérapeutique, l'expression et la reconnaissance de la honte comme appartenance subjective par le sujet signent qu'il n'y a pas perversion et constituent un point d'appui essentiel dans l'affirmation et la reconstruction de l'identité. Notre réflexion insiste, à travers une évocation clinique, sur cette co-occurrence entre traumatisme sexuel et sentiment de honte.
\end{abstract}

Mots clés: Identité, narcissisme, psychothérapie, sentiment de honte, trauma sexuel 


\section{Introduction}

Le fantasme est [...] tenu secret, son approche se signalant par un évanouissement subjectif enrobé de honte. L'amour est alors exigé de l'analyste comme une sorte de condition préalable à ce que le fantasme puisse être abordé, parce que seul le fait d'être aimé pourrait sauver la dimension subjective qui s'effondre dans la honte.

Serge André (1993, p. 53)

Ce travail est issu d'une réflexion élaborée, d'une part à partir de la déposition d'évènements traumatiques dans le cadre de psychothérapies d'enfants et d'adultes, d'autre part d'un travail de recherche en cours, mené par les deux auteurs de l'article, sur les circonstances subjectives et métapsychologiques de possibilité de développement par le sujet victime de traumatisme sexuel, d'une honte pathologique névrotique ou de stratégies défensives qui évoquent la perversion et dont l'absence/jouissance de la honte symbolise la dialectique.

Étymologiquement, "honte" vient du francique haunita, même radical que honnir (1080) qui signifie "mépriser", et revêt une pluralité d'acceptions:

1. Indignité qui inflige un déshonneur humiliant,

2. (1273). Sentiment pénible de sa bassesse, de son déshonneur, de sa confusion, de son abaissement devant les autres, ou simplement de son ridicule,

3. (1611). Sentiment de gêne, de malaise, provoqué par la timidité, la modestie, le manque d'assurance, la crainte,

4. Avoir du remords, être dégoûté de, être gêné de. 


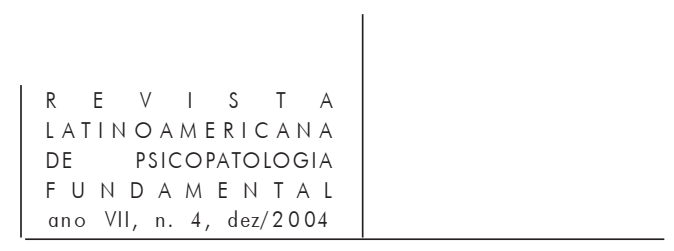

La tentative d'articulation de la honte au narcissisme va constituer le point de départ et d'appui de notre réflexion et plus largement, dans le cadre de la pratique clinique, sur la co-occurrence entre traumatisme sexuel et sentiment de honte. Autrement dit, comment entendre, au plan d'une approche plus métapsychologique, l'expérience de honte consécutive à un traumatisme sexuel? En ces zones extrêmes de l'expérience subjective, notre intérêt pour les modalités cliniques et théoriques de la honte dans ses aspects pathologiques et son traitement psychothérapique nous orientent vers l'exploration de son économie dans ses liens profonds avec le narcissisme, l'Idéal du Moi et le Moi-Idéal.

Dans cette configuration et ce sera ici le propos, le traitement psychothérapeutique des enfants ou des adultes victimes de traumatismes sexuels fait apparaître un sentiment de honte caractéristique. Pourquoi le traumatisme sexuel produit-il de la honte? Quelles insuffisances et quelles limites cette situation révèle, réactive, soulève? La première hypothèse ici envisagée prend en compte les deux instances psychiques de la personnalité que sont l'Idéal du Moi et le MoiIdéal, dont les éléments déterminants sont: d'une part le rapport de l'Idéal du Moi avec l'expérience d'une atteinte sexuelle et de la blessure narcissique irréversible qu'elle provoque, d'autre part le Moi-Idéal en rapport avec la déchéance ressentie dans la honte consécutive au traumatisme sexuel, honte de n'être que la chose, l'objet, le déchet de l'agresseur. La deuxième hypothèse postule que dans le dispositif psychothérapeutique, l'expression et la reconnaissance de la honte comme marque d'appartenance subjective par le sujet signent qu'il n'y a pas perversion et constituent un point d'appui essentiel dans l'affirmation et la reconstruction de l'identité. En effet, si, pour le sujet, l'expression de la honte est porteuse d'une acception précise, elle est pour le clinicien d'abord indice et messagère d'une signification particulière donnée à la parole. Il est vrai que l'usage de ce terme ne renvoie pas à un diagnostic, à une nosographie. Mais il mérite à s'expliquer sur le saut qui consiste à affirmer la présence de honte pour un sujet à partir de l'occurrence de ce terme dans son discours. Enfin, il y a lieu de situer la honte dans le transfert, dans l'adresse à un interlocuteur, ce que nous aurons l'occasion de développer à partir d'une étude de cas, plus précisément une situation qui évoque l'inceste et qui semble fournir une illustration de ce que nous appellerons communément honte pathologique névrotique.

La honte, qui empourpre le visage, est d'abord un sentiment social: elle apparaît le plus souvent en réaction au regard d'autrui et vient marquer l'échec de la confirmation narcissique. Cette primauté du regard dans l'expérience de honte, trouverait son hypothèse interprétative dans le fait que la honte prendrait sa source dans le regard de l'Autre, regard qui révélerait au sujet ses propres limites, son incomplétude, autrement dit dévoilerait le décalage insoutenable entre l'image narcissique de soi faite de perfection et une image reconnue dans le regard 


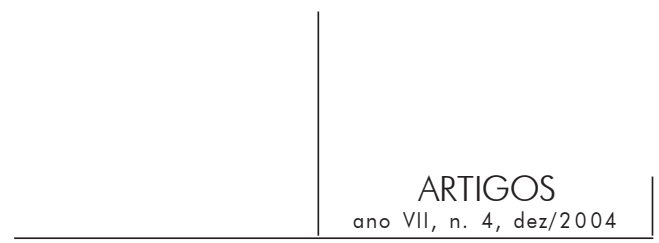

de l'Autre, d'insuffisance et d'imperfection. Nous nous situons là dans la perspective lacanienne classique qui postule, au sujet de l'Autre, que "[...] c'est de lui que le sujet se constitue, par quoi c'est de l'Autre que le sujet reçoit même le message qu'il émet" (Lacan, 1960, p. 807). La décharge fulgurante de honte telle qu'elle a été décrite chez Sartre dans une perspective phénoménologique, témoigne fort justement de cette expérience émotionnelle où une part du plus intime de soi se trouve brutalement exposé, véritable mise à nu du sujet qui le confine dans un chaos narcissique. Dans l'expérience de honte, l'effet de transparence ressenti en est la traduction la plus explicite, qui ne saurait mieux s'exprimer que dans l'expression langagière, assez coutumière, de mise à $n u$. Ce ravalement à une fonction d'objet engendre un grand désarroi chez le sujet, car il est alors en proie à une incertitude narcissique angoissante. En ce sens, la honte constitue une expérience subjective singulière, celle d'un sujet brusquement dévoilé dans son intimité de pensée, par la présence d'un regard dont il est impossible de se dérober. En tant qu'affect, la honte est d'emblée sociale, étroitement liée au regard de l'autre, tout autant qu'expression de la manifestation d'une revendication singulière.

Ici, il faut s'arrêter un instant sur la notion d'affect dans l'œuvre freudienne, l'auteur proposant une première classification des névroses selon la manière dont un sujet se comporte au regard des affects. Dans son article sur "L'Inconscient" (Freud, 1915), il définit l'affect de cette façon: “... les affects et sentiments correspondent à des processus de décharge dont les manifestations finales sont perçues comme sensations" (p. 84). Plus précisément, l'affect est défini comme la traduction subjective de la quantité d'énergie pulsionnelle et de ses multiples variations. Enfin, le concept d'identification est défini comme une opération par laquelle le sujet humain se constitue: l'identification aspire à “... rendre le moi propre semblable à l'autre pris comme modèle" (Freud, 1921, p. 169). Ce concept d'identification se trouvera enrichi par différents apports, dont celui de narcissisme, que Freud introduit dans le texte intitulé "Pour introduire le narcissisme" (1914) en envisageant tout particulièrement les investissements libidinaux. Le souci majeur de Freud est de mettre en évidence le narcissisme comme une forme d'investissement pulsionnel nécessaire à la vie subjective, comme une donnée structurale du sujet. Il représente "... une sorte d'état subjectif, relativement fragile et facilement menacé dans son équilibre" (Chemama, 1993, p. 200). Ce bref rappel du rôle du narcissisme dans la psychogenèse de l'enfant montre que chaque être investit son moi grâce aux investissements dont il a pu être l'objet dans les premières années de sa vie.

Lacan a mis en rapport ce moment inaugural de la formation du moi avec l'expérience narcissique fondamentale qu'il désigne sous le nom de stade du miroir, qui marque une étape génétique et ontologique fondamentales, dans la 


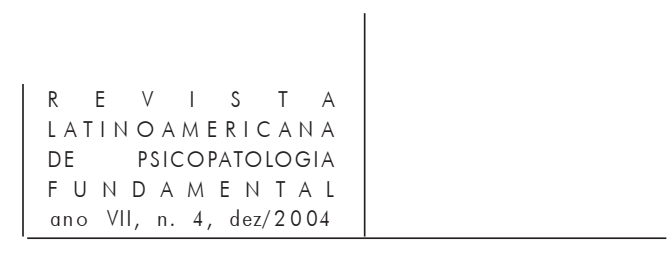

mesure où va s'y constituer la première ébauche du moi. Face au miroir, l'enfant va anticiper, dans un leurre spéculaire et imaginaire, l'appréhension et la maitrise de son unité corporelle: "Il y suffit de comprendre le stade du miroir comme une identification au sens plein que l'analyse donne à ce terme: à savoir la transformation produite chez le sujet, quand il assume une image" (Lacan, 1945, p. 94). Cette expérience narcissique fondamentale constitue la matrice symbolique de ce qui sera le moi. Elle est au fondement de l'expérience imaginaire du moi, constitué d'emblée comme "je-idéal" et "souche des identifications secondaires". (ibid.) En ce sens, la relation intersubjective, qui est marquée des effets du stade du miroir, est d'abord une relation imaginaire et duelle, où le moi est constitué comme un autre et autrui comme alter ego.

Le registre imaginaire représente, avec le réel et le symbolique, l'un des trois registres fondamentaux du champ psychanalytique lacanien. Dans une dimension intrasubjective, l'imaginaire concerne le rapport fondamentalement narcissique du sujet à lui-même, rapport où “... l'individu humain se fixe à une image qui l'aliène à lui-même, c'est là l'énergie et c'est là la forme d'où prend origine cette organisation passionnelle qu'il appellera son moi" (Lacan, 1948, p. 113). Pour Lacan, toute relation imaginaire est vouée à ce leurre: "Je est un autre" (ibid., p. 118) écrit-il reprenant le poème de Rimbaud. L'édification de l'idéal du moi ou $d u$ moi-idéal en tant qu'instance imaginaire représente l'instance de la personnalité qui, dans une dimension symbolique, a la charge de réguler la structure imaginaire du moi et la panoplie des identifications qui s'y déploient, ainsi que l'ensemble des conflits qui animent et régissent, dans une dynamique intersubjective, les rapports du sujet à ses semblables.

Le registre du réel ne peut être défini que par rapport au symbolique et à l'imaginaire. Il se caractérise d'un défaut fondamental: il est inconnaissable et non symbolisable. La honte participe de ce registre, elle se spécifie de sa rencontre avec le réel. C'est là qu'elle s'éprouve, dans son lien à l'originaire et dans le rapport scopique à l'autre semblable: le réel saisi dans le regard d'autrui. Le sujet honteux tombe, comme déchet, sous le regard de l'autre, il se fait alors - au sens lacanien - objet a, objet cause du désir qui répond à cette place de la vérité, ici dans la honte qui est l'épreuve de son dévoilement, en tant que le sujet le présentifie au regard de l'autre. La honte, c'est du pur réel, c'est-à-dire qu'elle surgit de cette réalité qui n'est pas ordonnée par le symbolique. Dans l'expérience de honte, la mise à nu du sujet sous le regard d'autrui invoque ce réel, par où "[...] il revient dans la réalité à une place où le sujet ne le rencontre pas, sinon sous la forme d'une rencontre qui réveille le sujet de son état ordinaire" (Chemama, 1993, p. 278). Les manifestations physiques, corporelles et physiologiques de la honte témoignent de cette effraction qui fait la chute du sujet dans un réel non symbolisable, signant du même coup l'échec de toute symbolisation. 


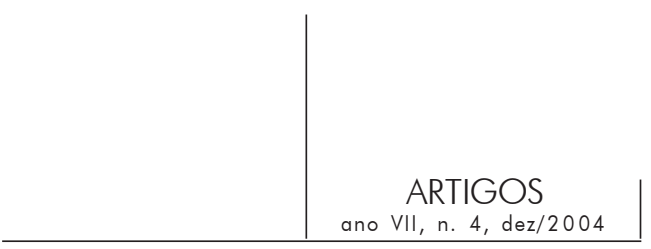

Le registre symbolique marque l'affranchissement, chez l'enfant, de la capture imaginaire dans laquelle il se trouvait, depuis l'origine, inscrit. Au temps pré-spéculaire où l'enfant se vit comme morcelé, succède le stade où la constitution du moi s'unifie dans la dépendance d'une identification aliénante à l'image spéculaire et en fait le siège de la méconnaissance. Mais pour que le petit homme puisse s'approprier et intérioriser cette image, l'Autre (incarné par la mère) doit le reconnaître et le confirmer dans son existence de sujet. Ce signe de reconnaissance va fonctionner comme trait unaire, ${ }^{1}$ c'est-à-dire comme signifiant à partir duquel va pouvoir se constituer l'identification symbolique et l'ébauche de l'idéal du moi.

À l'appui de ces considérations et dans une perspective psychanalytique, nous dirons que la honte comme éprouvé narcissique est en lien avec l'Idéal du Moi et le Moi-Idéal. Le travail psychothérapeutique auprès de sujets souffrant de honte montrent "l'état singulier de déficit narcissique" (Jacobi, 1998, p. 25). Dans ce sens, la clinique de la honte concerne directement le narcissisme et plus précisément la honte pourrait relever d'un fonctionnement sur le mode narcissique. C'est à partir d'une évocation clinique que va être mis à l'épreuve ce corpus d'hypothèses. Mais au préalable, nous proposons de resituer d'abord l'apparition de la honte dans la théorie freudienne.

\section{Métapsychologie de la honte dans l'œuvre freudienne}

En un sens large, le mot allemand Scham désigne dans l'usage freudien, aussi bien la honte comme formation réactionnelle dans la névrose obsessionnelle, comme "digue psychique" avec le dégoût et l'exigence d'idéal esthétique et moral, et dont la mission est de faire rempart à l'envahissement pulsionnel, notamment des pulsions sexuelles de voyeurisme et d'exhibitionnisme au début de la période de latence. Enfin, dans le cadre de la seconde théorie de l'appareil psychique, le dégagement de la notion de sentiment de culpabilité devait conduire Freud à opérer une distinction théorique avec le sentiment de honte, et à spécifier le rapport dialectique entre ces deux notions. L'histoire de la pensée freudienne est complexe et l'étude des textes ne permet pas de localiser une acception du concept de honte précise et déterminée, dans la mesure où le terme allemand est tantôt traduit par

1. Le trait unaire est "Un concept introduit par J. Lacan, à partir de S. Freud, pour désigner le signifiant sous sa forme élémentaire et pour rendre compte de l'identification symbolique du sujet”. Ibid., p. 334. 


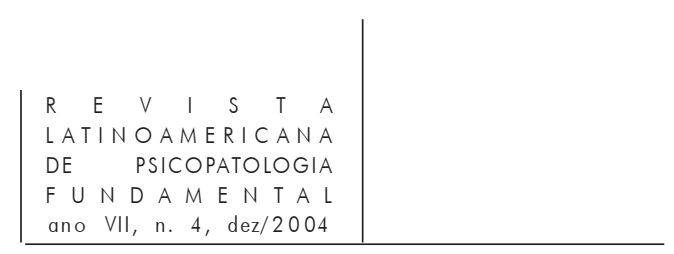

"honte" et tantôt par "pudeur", deux équivalents sémantiques qui ne permettent pas de conclure à un usage absolument univoque.

Dès ses premiers écrits, Freud (1905) assimile la honte à l'action des forces refoulantes - répressives - qui ont pour visée de lutter contre le surgissement des pulsions. Dans plusieurs textes, l'auteur cite simultanément “... le dégoût, la honte, la moralité" (p. 101). Ce qui était initialement objet de plaisir - plaisir directement lié à la dimension sexuelle sous-jacente - devient, sous l'effet du refoulement et des formations réactionnelles, objet de pudeur, de dégoût ou de honte. Dans son article intitulé "Nouvelles remarques sur les psychonévroses de défense" (1896), Freud écrit: "Alors un reproche s'attache au souvenir de ces actions génératrices de plaisir; la relation avec l'expérience initiale de passivité permet - souvent, seulement après des efforts conscients dont le sujet se souvient - de refouler ce reproche et de le remplacer par un symptôme primaire de défense. Scrupulosité, honte, méfiance de soi-même, sont les symptômes qui ouvrent la (...) période de santé apparente mais en fait de défense réussie" (p. 67).

Mais c'est surtout avec la seconde topique que s'élabore le positionnement métapsychologique de la genèse du sentiment de honte. Dans "Psychologie des foules et analyse du moi" (1921), Freud reconnaît au Surmoi ("Über-Ich") les fonctions suivantes: “... l'auto-observation, la conscience morale, la censure onirique et l'exercice de l'influence essentielle lors du refoulement" (p. 173). C'est le Surmoi qui donne le sentiment d'être surveillé et épié par une partie de soi-même, contribuant ainsi au caractère paranoïde du Moi. D'un point de vue métapsychologique, la honte est subordonnée à l'Idéal du Moi ("Idéal-Ich") dans la mesure où elle est le reflet du conflit intra-psychique entre le Surmoi et le Moi, conflit entre les instances psychiques qui est déterminant pour le sujet. Dans "Le moi et le ça" (1923), l'auteur nous propose la formule suivante: "Tandis que le moi est essentiellement représentant du monde extérieur, de la réalité, le sur-moi se pose en face de lui comme mandataire du monde intérieur, du ça” (p. 249). C'est ainsi que l'éclosion des conflits entre l'instance moïque et l'Idéal du Moi vont inaugurer l'opposition entre monde extérieur et monde intérieur, entre réel et psychique dans un conflit inter-instances où les exigences du Surmoi despotique vont tyranniser le Moi, surévalué négativement par rapport à l'Idéal du Moi coercitif. Dans le vécu de honte se produirait ainsi une coalescence entre monde intérieur et monde extérieur: le Moi se trouve pris dans un double mouvement identificatoire entre d'une part le Surmoi qui juge et auquel il se réduit et d'autre part l'identification imaginaire de sujet "honteux" imputée au regard d'autrui. Une dialectique conflictuelle dynamique s'instaure entre le Surmoi et l'Idéal du Moi (comme figure surmoïque) qui s'effondre, pris dans les rets de cette instance "cruelle" qui le critique et par ailleurs point de mire du regard d'autrui, témoin de la fragmentation et du ratage de sa confirmation narcissique. 


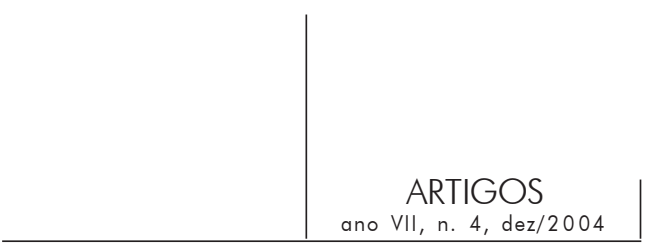

Dans le même texte, Freud introduit la notion de Moi-Idéal ("Ich-Idéal") mais il faut souligner que parfois, avec l'Idéal du Moi et le Surmoi, ces trois termes sont utilisés de manière synonymique dans l'œuvre freudienne, ne permettant que difficilement leur délimitation sémantique et même métapsychologique. En effet, l'auteur admet l'existence d'une différenciation, au sein du Moi, d'une instance qu'il nomme indifféremment Idéal du Moi ou Surmoi, issu du système perception-conscience, comme ses propos en témoignent: "... l'idéal du moi ou sur-moi [est] la représentance de notre relation aux parents" (Freud, 1921, p. 248). Le Surmoi est au centre de la question morale. Il a un rôle de juge et de censeur à l'égard du Moi. Il inhibe les actions du sujet, induit le remords, les scrupules et le repentir. Il est "la voix de la conscience", "la grosse voix" impérative qui réclame obéissance et soumission. Le Moi-Idéal, lui, caractérise le moi réel, objet convoité des premières gratifications et satisfactions narcissiques. Et ce n'est pas dénué de nostalgie que le sujet aspire à retrouver ce Moi-Idéal conçu comme un idéal de complétude narcissique, édifié sur le modèle du narcissisme infantile: "Nous sommes au centre de tout désir de restaurer une toute-puissance à laquelle nous pensons et croyons avoir renoncé". (Jacobi Tout ceci s'inscrit dans un rapprochement théorique que Freud opère entre ces deux instances du Moi-Idéal et de l'Idéal du Moi, leur attribuant conjointement les mêmes fonctions d'interdiction, de censure et d'idéalisation.

Pour Lacan (1960), le moi idéal s'élabore à partir de l'image du corps propre dans le miroir, image qui constitue le support de l'identification primaire de l'enfant à l'autre semblable: "C'est cette image qui se fixe, moi idéal, du point où le sujet s'arrête comme idéal du moi. Le moi est dès lors fonction de maîtrise, jeu de prestance, rivalité constituée" (p. 809). Ainsi, le sujet assume une certaine image de lui-même, enrichie des processus d'identification en perpétuelle mouvance, mais en aucun cas il ne peut se réduire à un champ exclusivement spéculaire de ce qu'il en est de son identification au miroir, car c'est toujours par la médiation de l'Autre, par le regard porté sur lui, que le sujet se perçoit et s'appréhende.

$\mathrm{Au}$ sens freudien, la culpabilité concerne le Surmoi. Alors que la honte, en tant qu'éprouvé narcissique, est directement liée à l'Idéal du Moi. Elle implique une mise en défaut de soi, devant témoin. Dans le vécu de honte, l'Idéal du Moi défaille en regard des énoncés impératifs surmoïques et en regard de l'Autre aussi, témoin de ces turpitudes et de sa déchéance. En ce sens, l'inscription de la honte dans le circuit pulsionnel du regard s'accompagne de cette épreuve intersubjective du rapport du sujet à l'Autre, altérité radicale qui ne se résorbe pas, Autre qui n'est pas un semblable.

Dans une perspective classique, la métapsychologie de la honte dans l'œuvre freudienne rend compte de la différence du Surmoi comme instance critique et punitive à l'égard du Moi, et introduit la honte comme relation entre les différentes 


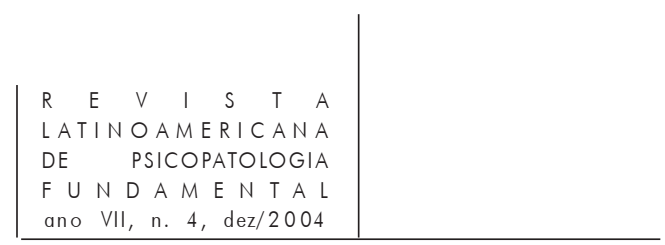

instances de la personnalité (Moi - Surmoi - Idéal du Moi) au sein de l'appareil psychique.

En ce sens, le modèle prototypique freudien rend compte de la honte dans son rapport exclusif au refoulement du sexuel, selon l'hypothèse phylogénétique, le mythe de la Genèse inaugurant la naissance du sentiment de honte, qui passe par le regard sur "les parties honteuses" et l'introduction de la différence des sexes. La démarche de cette réflexion clinique et d'élucidation théorique ouvre par ailleurs la voie à une articulation de la honte au Moi-Idéal, référence à une complétude imaginaire qui constitue l'objet même de l'intérêt et de l'amour narcissique, en référence à une représentation de soi et de l'épreuve que représente le fait de se reconnaître imparfait, limité, manquant, c'est-à-dire soumis à l'épreuve de la castration. Le surgissement de la honte ne porterait donc pas seulement sur le sentiment de valeur et d'estime de soi mais aussi et surtout sur le sentiment d'identité, dont la honte en révèlerait les fissures et les ruptures. Dans cette perspective, la honte concerne directement le Moi-Idéal et manifeste la mise en échec, le ratage d'un idéal de toute-puissance narcissique. L'identification grandiose par laquelle le sujet essaie de reconquérir l'omnipotence infantile passée est brutalement mise à jour dans son achoppement même, ce que la honte révèle. Tout ceci vient en quelque sorte provoquer une réflexion quant à la conceptualisation de la honte dans le cadre de la seconde topique de l'appareil psychique et plus largement dans le champ de la psychopathologie freudienne. La difficulté de conceptualisation d'une métapsychologie de la honte - hypothèse théorique fondamentale - réside dans la prise en compte incontournable de cet objet qui est le regard de l'autre puisque la honte est d'emblée un sentiment social. Comme l'écrit C. Barazer (2000), "En ce sens la honte pour être pensée nécessiterait une sorte de topique limite, à la limite de l'intra et de l'intersubjectif. Peut-être est-ce là une des difficultés à la penser méta-psychologiquement" (p. 17). Il conviendra de revenir sur cette conceptualisation.

2. En se verticalisant, l'être humain met à découvert ce qui était maintenu caché, "recouvert" [gedeckt], dans la position courbée. D'où l'association de ces deux termes: les organes génitaux deviennent solidairement sichtbar und schutzbedürftig (visibles et exigeant une protection). Apparaît ainsi le fait d' "avoir honte" [das Schämen], ce terme pouvant aussi être traduit par "pudeur". Schneider, M. (1996). "Le franchissement du seuil, Freud et la thématique du regard ", p. 22. 


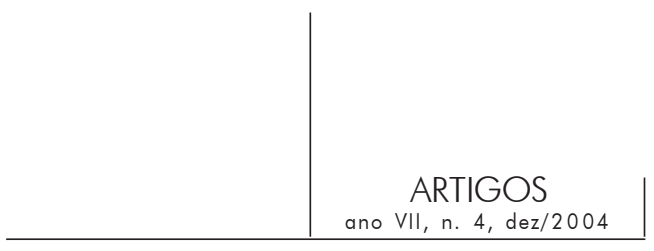

Étude de cas

Préambule

La patiente ${ }^{3}$ dont nous exposerons maintenant quelques fragments d'histoire nous est adressée pour une situation assez dramatique d'inceste familial. Les entretiens psychothérapeutiques avec Madame M. s'étendent sur plusieurs mois; l'ensemble des observations cliniques issues de ce suivi débouchent sur les axes de réflexion suivants:

1. L'idée de plaisir énoncée sous une forme de dénégation,

2. La honte signe qu'il n'y a pas perversion,

3. L'idéal esthétique comme contre-investissement de la honte,

4. Le traitement psychothérapeutique de la honte, dans la succession des différentes temporalités psychiques et de leur étroite combinaison, passe par les trois temps suivants: l'inhibition, la mise en mots de la honte, l'affirmation et la reconstruction de l'identité.

Éléments d'anamnèse

Madame M. est une jeune femme de 36 ans, d'aspect soigné et d'apparence plutôt masculine, les cheveux courts, le teint mat et les yeux marrons.

Sa demande d'hospitalisation dans un service psychiatrique repose sur un état dépressif récurrent (qui s'est aggravé depuis la naissance de son fils) et sur une tentative de suicide par phlébotomie et ingestion médicamenteuse. La patiente décrit un pessimisme, un sentiment d'insatisfaction, une incapacité à assumer ses responsabilités, une anxiété généralisée avec des plaintes somatiques multiples. Elle évoque également une violence contenue depuis plusieurs années, une intolérance vis-à-vis de son entourage affectif et surtout de son enfant, une asthénie, une atteinte des conduites instinctuelles telles que des troubles du sommeil, des cauchemars fréquents, une perte de l'appétit et du désir sexuel.

Mme M. vit en concubinage avec son compagnon depuis une dizaine d'années. Ils ont un fils, Lucas, âgé de quatre ans. Mme M. est salariée dans une entreprise de manutention et son conjoint est employé de mairie. Au niveau de la fratrie, Mme M. est la cadette d'une fratrie de quatre: elle a trois sœurs.

3. La situation rapportée concerne l'un des auteurs de cette recherche (Delphine Scotto Di Vettimo). Son élaboration après-coup a été faite par les deux signataires de l'article. 


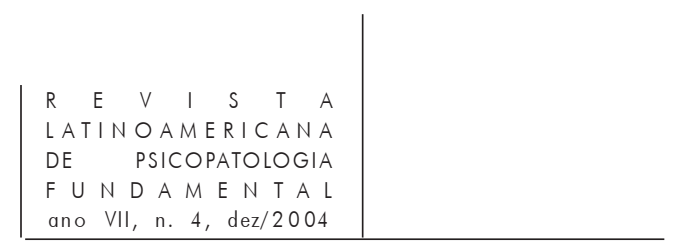

Au niveau des antécédents, le dossier de soins mentionne "trois tentatives de suicide par ingestion médicamenteuse à l'âge de 12, 13 et 14 ans, la dernière tentative ayant nécessité un long séjour dans un service de réanimation”. Par ailleurs, les éléments historiques recueillis témoignent en faveur d'un inceste familial durant l'enfance.

\section{Récits cliniques}

\section{L'inhibition}

À notre premier entretien, Madame M. exprime d'emblée des sentiments très profonds de dévalorisation qui seraient à la source de ses idées suicidaires et qui révèlent une fragilité narcissique. Très rapidement, elle va évoquer les événements traumatiques survenus dans sa vie, à savoir l'inceste familial dont elle et ses sœurs ont été victimes durant leur enfance: "J'ai eu une enfance plutôt noire avec des parents incestueux. J'ai perdu ma virginité avec mon père, on faisait l'amour comme mari et femme, comme si on était marié. À l'adolescence, je suis tombée enceinte de mon père... je me sens sale dans mon corps". Curieusement, son discours apparaît dénué d'affects et d'émotions, en total décalage avec la gravité de son contenu. Elle rapporte son état dépressif à ces abus sexuels incestueux, dépression particulièrement réactivée depuis la naissance de son fils, et surtout sa crainte de reproduire sur lui ce qu'elle-même a subi: "Tout allait bien jusqu'au moment de ma grossesse”. Aujourd'hui, Lucas a quatre ans; elle évoque un fait récent où, alors qu'il était insupportable, elle lui a mis une fessée: “J'ai peur de devenir comme mon père, je culpabilise d'avoir frappé mon fils, j'ai cherché à lui faire mal'. Elle précise que quatre ans, c'est l'âge où son propre père a commencé à être violent à son égard. Je remarque que ces propos dénotent par moments une réelle difficulté à faire la part de sa propre identité face à celle de son enfant.

Concernant son couple, la patiente décri "une vie affective stable et banale" ainsi qu'une bonne entente avec son compagnon jusqu'à la naissance de leur fils, qui correspond au début de ses troubles dépressifs. Il semble qu'elle pourrait évoquer ici une sorte de déception d'une vie ordinaire, peu conforme à son Idéal du Moi et à son Moi-Idéal. De son compagnon, elle dira: "C'est un homme aimant mais avec lequel la relation est devenue insatisfaisante... je ne supporte plus qu'il me touche, je suis devenue très agressive avec lui". Puis elle revient sur les maltraitances subies. Elle décrit son père "violent et alcoolique, l'ayant menacée de mort ainsi que le reste de la famille”. Elle se dit très marquée par l'agressivité et la violence paternelles. Quant à sa mère, elle la considère comme complice du 


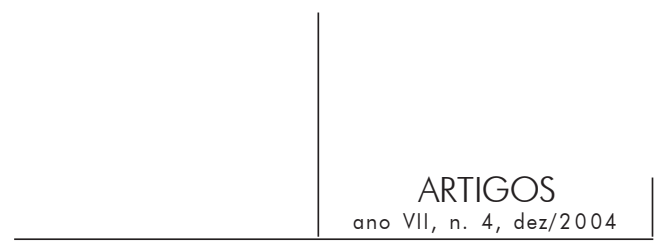

père et à la fois victime, comme ses filles. Au sujet du décès paternel survenu six ans plus tôt, Mme M. affirme qu'il ne lui a causé aucun chagrin et qu'en outre, elle n'a pas assisté à ses funérailles: "Mon père a cassé quelque chose en moi. Je le hais. Sa mort m'a laissée indifférente” dit-elle. Dans la constellation familiale prédominait ainsi la toute-puissance d'un père totémique au sens freudien, tel qu'il est décrit dans Totem et tabou (1912), figure paternelle qui se situe à la fois du côté de la loi et du dérèglement de cette loi, à la fois du côté du désir et de la jouissance. Ici, les relations familiales semblaient marquées d'une dialectique binaire dominant/dominé excluant de fait toute référence à la triangulation et à l'individuation. La violence et la perversité du père se manifestaient par des menaces de mort (visant entre autres, à maintenir le secret de l'inceste) entretenant ainsi un climat d'insécurité et de terreur permanentes. Investi de l'illusion d'un pouvoir suprême, le père s'arrogeait un droit de vie et de mort sur sa famille, sa violence physique et verbale venant "bâillonner" toute tentative d'opposition et assujettir femme et enfants à son désir et à sa barbarie. Comme le souligne J. Clavreul (1987), “... le pervers n'a pas réussi à éprouver sa solidité (de la loi) en la rattachant à son origine dans la différence des sexes et de l'interdit de l'inceste" (p. 199). Ce huis-clos incestueux durera des années, aucun membre de la famille n'ayant jamais révélé ces abus. Enfin, l'autre forme de violence éprouvée par la patiente est liée à la mère - complice active - qui, dans un déni massif des faits, faisait directement écho à la violence du père abuseur.

Durant une première période, Mme M. pleure beaucoup durant les entretiens. Elle évoque à plusieurs reprises l'inceste familial dont elle a été victime, et surtout sa culpabilité liée au fait de ne pas s'être opposée à ces actes: "Je regrette de ne pas avoir dit 'non' à mon père, je m'en veux, je me dégoûte moi-même”. Murée dans une culpabilité massive, ces paroles ne semblent à aucun moment se soulager d'être dites, ce qui nous amène à supposer que le problème est à situer ailleurs, c'est-à-dire du côté de la honte de cet inceste. De la récurrence des autoreproches va progressivement se déployer, dans l'espace transférentiel, un flot d'injures à l'encontre du père. Ce qui importe, c'est qu'elle puisse enfin déposer - dans le site psychothérapique - l'outrage extrême d'avoir été contrainte à des rapports sexuels avec son père, sa mère et ses sœurs. Un jour, elle dit: “J'aimerais être moi, enlever toutes ces choses dégoûtantes de mon esprit... je voudrais aller au cimetière pour dire à mon père tout ce que je ressens, tout ce qu'il m'a fait subir". De “dégoût”, de “choses dégoûtantes”, il en sera beaucoup question durant ces premières semaines, mais le mot "honte" ne sera jamais énoncé. Autrement dit, elle ne peut mettre en circulation cette honte dans le transfert qu'à la condition de la dire sous une forme travestie, le dégoût venant la désigner et exprimer directement le rapport au corps, "le dégoût d'avoir fait ça". 


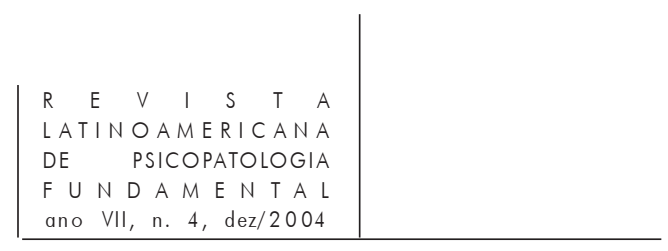

La mise en mots de la honte

Alors que la patiente avait évoqué, au début de la prise en charge, un vécu de culpabilité lié à sa "passivité" dans l'inceste familial, c'est un vécu de honte qui va bientôt occuper les séances.

Nous en sommes au douzième entretien. Mme M. a le visage tendu. Elle se triture les mains, semble embarrassée. Elle finit par dire, dans l'évitement de la rencontre de nos regards: "Les images de l'inceste se déroulent comme un film permanent... je n'arrive plus à laisser le passé derrière moi... il m'obsède”. À cette évocation, son regard se brouille et des larmes coulent de ses yeux où on pouvait lire un grand désarroi. Non sans une grande confusion émotionnelle, elle parviendra juste après à confier: "Je me sens coupable et honteuse de ce que j'ai fait". S'ouvrent ainsi les prémices d'une mise en récit de sa honte et de sa crainte conjointe de mon jugement négatif à son égard: "J'ai honte de moi. J'ai peur que vous ayez honte de moi et que vous pensiez que j'avais du plaisir dans ses relations incestueuses! D'ailleurs, je ne peux pas vous regarder en face quand je vous dis cela! J'ai trop honte! Je crains de voir dans vos yeux et votre regard un jugement”. Effectivement, à cette évocation, son regard balaye la pièce de gauche à droite, en évitant de rencontrer le mien. Un silence s'installe. Face à ce que je ressens comme une profonde détresse et un besoin d'étayage, j'essaie de la rassurer et je mets en mots à quel point ce doit être difficile pour elle de pouvoir aborder ces événements traumatiques de son enfance. C'est par ce biais que l'on pourrait rapprocher ici la honte comme épreuve du dévoilement du sujet étroitement lié au regard de l'autre, comme S. André (1993) l'indique lui-même: "On sait que le névrosé se tait sur son fantasme, ou qu'il ne le livre, dans l'expérience analytique, qu'avec la plus grande peine, comme un aveu arraché à la honte (...). C'est que, pour lui, faire passer le fantasme de la scène privée à la scène publique en le confiant à un auditeur, c'est automatiquement se désigner comme coupable et s'exposer aux foudres de l'Autre" (p. 54).

C'est à partir du moment où elle aura pu confier être victime de honte, avoir honte de cet inceste et ne pas oser affronter mon regard de crainte d'y lire un jugement, qu'elle va commencer à détailler les violences sexuelles intra-familiales subies de l'âge de huit ans jusqu'à l'adolescence. Autant dire que c'est à partir de la nomination de la honte et de l'idée de plaisir énoncée sous une forme négative qu'elle va pouvoir aborder le contenu des scènes incestueuses. Dans les semaines qui suivront, elle décrira, de manière itérative, les maltraitances subies, répétition qui manifeste une tentative d'élaboration psychique et de (ré)appropriation subjective. Après le dépassement de "la honte de la honte", Mme M. essaie de dépasser et de métaboliser les souvenirs de son enfance qui envahissent son présent et qui prennent figures d'obsessions. Elle relate le début 


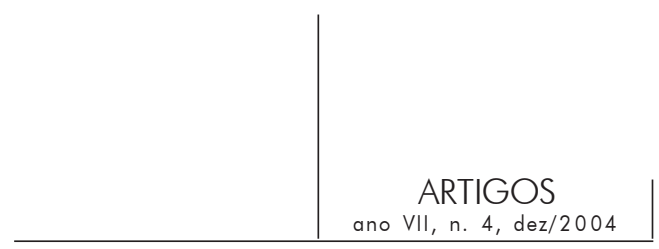

des violences physiques paternelles à l'âge de quatre ans et les premiers attouchements sexuels à l'âge de huit ans. Les révélations qui vont suivre dépassent toute possibilité de mise en représentation, tant elles mettent en relief l'horreur de la transgression maternelle et paternelle de la Loi de l'interdit de l'inceste: "Les attouchements sont devenus de plus en plus intimes... puis mon père a commencé à me faire des pénétrations orales, vaginales puis des sodomies. Rapidement, il a demandé à ma mère et mes sæurs de participer. Puis il a décidé de nous filmer pendant les actes sexuels". Cet énoncé des actes barbares subis "forçait" littéralement l'oreille de la psychologue par sa monstruosité, sa violence mais aussi et surtout par la volonté totalitaire qu'il impliquait. Nous citerons ici E. Blanc (1997) lorsqu'elle écrit: “Dans l'inceste, ce n'est pas l'acte en lui-même qui fait horreur, mais ce à quoi il renvoie, c'est-à-dire à la transgression des limites que l'homme s'est donné pour signifier son humanité, limites établies dans la parole et par la parole". Cet au-delà de l'horreur et des limites, Mme M. l'évoquera d'elle-même lorsqu'elle dira, à un moment de l'entretien, que durant les actes sexuels, ce n'est pas un homme mais "une bête qui abusait d'elle" et qu'elle énoncera, non sans manifestations émotionnelles, le sadisme itératif de ce dernier à son encontre. Dans les faits rapportés, il semble qu'elle était en position d'objet littéralement "sadisé" par son père, dont les conduites pathologiques et les alcoolisations quotidiennes étaient vécues par la patiente comme la menace perpétuelle d'un risque d'anéantissement psychique et même physique. Elle dit s'être défendue de toutes ses forces, mais explique son impuissance à se protéger, se sentant sous son emprise et n'avoir pu faire face à ses avances perverses.

Six mois séparent aujourd'hui la séance qui suit du premier entretien. Mme M. décrit une dégradation importante des relations conjugales dans le sens où elle revit dans l'intimité de son couple la relation incestueuse: "Je ne supporte plus que mon mari me touche car je vois mon père à sa place”. Ainsi, la réactualisation des évènements traumatiques dans le transfert avait pour corrélat une honte empreinte de sentiments incestueux qui venait laminer et déliter sa vie sexuelle d'adulte. Elle évoquera en outre une accentuation de phobies sociales en ces termes: "J'ai beaucoup de mal à être confrontée au regard des gens" et décrit également une recrudescence d'idées morbides qui prennent l'allure de phobies d'impulsions suicidaires. La patiente se décrit attirée et extrêmement angoissée par des objets tranchants qui provoquent des ruminations morbides de scénarios de passage à l'acte suicidaire.

Par ailleurs, elle manifeste beaucoup d'inquiétude au sujet de son fils du fait d'une aggravation des impulsions de violence ressenties à son égard: "Je me fais peur par rapport à cette violence”. En outre, il convient de noter l'accentuation de rituels de lavage lors des permissions le week-end, en particulier des rituels 


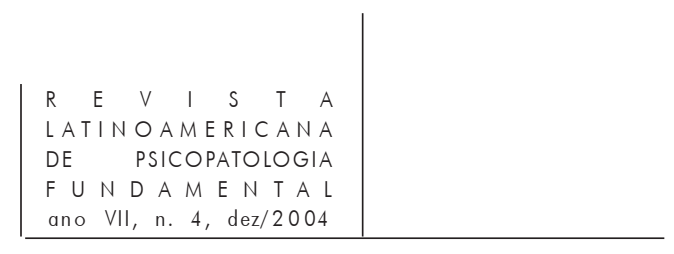

concernant la propreté des toilettes et des vitres: "Je ne supporte pas de voir la moindre trace" répète-t-elle. Enfermée dans ses souvenirs inoubliables, elle se demande comment supporter cette réalité ressentie comme intolérable, celle de sa propre histoire. En même temps, en me parlant de ces images qui s'imposent à elle en permanence, elle révèle que quelque chose ne peut et ne pourra jamais se transmettre, à savoir une expérience dont les mots s'avèrent impuissants à la soutenir, la représenter, l'expliquer. Et c'est bien de l'horreur du trauma dont il s'agit. C'est cela aussi qui est informulable.

Dans les derniers entretiens qui précéderont sa sortie, elle évoque ses trois tentatives de suicide à l'adolescence. Voici ce qu'elle en dit: "J'ai fait ma première tentative de suicide à l'âge de 12 ans, la deuxième un an plus tard... j'ai failli mourir pour de bon. Je regrette que l'on m'ait ramenée à la vie". Le dernier passage à l'acte suicidaire faisait suite à la grossesse issue des rapports sexuels incestueux. Mme M. avait alors 14 ans. Elle subira une Interruption Volontaire de Grossesse (I.V.G.) et menace, dès son retour au domicile, de tuer son père si les abus sexuels persistent.

De l'âge de 14 ans à 18 ans, elle subira encore des violences physiques et verbales quotidiennes mais les menaces proférées seront suivies d'effets puisque les abus sexuels cessent: "Je me dégoûte moi-même d'avoir participé à ces partouses familiales. Mon père prenait des photos et nous filmait... il faisait des films pornographiques".

Enfin, c'est lors de ce même entretien qu'elle affirmera avoir vécu plusieurs aventures homosexuelles précédant sa rencontre avec le père de Lucas. Elle confiera également avoir fréquenté des clubs d'échangisme et participé à "des partouses" à l'initiative d'une partenaire de même âge, décédée accidentellement juste après leur séparation. Elle soulignera n'avoir aucun regret de ces expériences qui lui ont permis de mieux accepter son passé. Enfin, elle fera part de la persistance "de pulsions homosexuelles" qui mettent sérieusement en question la poursuite de sa relation avec son compagnon. Voici ce qu'elle dira: "De toutes façons, si je quitte mon ami, ce ne sera plus avec un homme mais avec une femme que je referais ma vie". Dans la mise en avant par la patiente elle-même du terme "homosexualité", quel sens subjectif revêtait ce terme?

Esquisse d'une reconstruction narcissique et idéal esthétique

Au terme de la prise en charge, Mme M. affirme ne pas souhaiter reprendre son activité professionnelle de manutentionnaire qu'elle décrit pénible et sans intérêt. Elle émet le projet d'un reclassement professionnel: "Je me suis renseignée pour suivre une école d'esthétique”. Nous émettrons deux hypothèses sur 


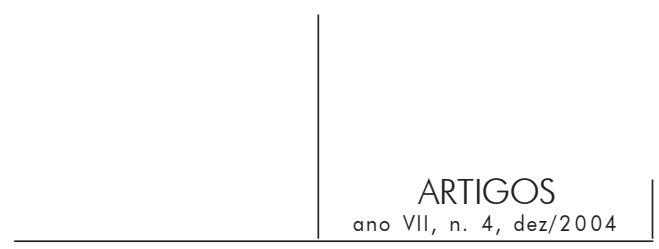

le sens subjectif de ce choix, qui ouvrent la question du devenir de la honte et des stratégies de réparation du sujet. D'une part, le vécu narcissique de l'atteinte physique et psychique motiverait ce souhait de réorientation professionnelle: investir dans l'esthétique témoigne du désir de trouver une image dans laquelle se reconnaître et s'aimer. Pour la patiente, il s'agirait de retrouver une identité subjective et de réhabiliter cette mauvaise image qu'elle a d'elle-même. En ce sens, devenir esthéticienne viserait implicitement à redéfinir les contours d'une identité, avec l'espoir que la nouvelle image socioprofessionnelle influencera l'identité subjective, l'image que la patiente a d'elle-même. ${ }^{4}$ D'autre part, à la lumière des conceptions théoriques freudiennes, la préoccupation esthétique traduirait un contreinvestissement de la honte. Dans les Trois essais sur la théorie sexuelle (1905), Freud évoque les "forces psychiques" (p. 99) qui vont lutter et s'opposer à la pulsion sexuelle et qui, "telles des digues, resserreront son cours (le dégoût, la pudeur, les aspirations idéales esthétiques et morales)" (ibid.). Cette honte - hormis la fonction de symptôme qu'elle revêt dans la névrose obsessionnelle - est définie ici comme "formation réactionnelle" avec le dégoût et l'exigence d'idéal esthétique et moral, dont la visée est de faire "digue" à l'envahissement et l'assaut pulsionnel, en particulier des pulsions sexuelles de voyeurisme et d'exhibitionnisme de la période de latence. Cette théorisation montre le caractère de proximité de la honte avec la revendication pulsionnelle qu'elle est chargée de contenir et d'autre part son aspect primaire et brut. Dans cette acception, l'idéal esthétique témoignerait d'une ébauche de métaphorisation de la souillure et de la tâche.

\section{Réflexion théorico-clinique}

L'analyse de ce fragment clinique permet à présent de revenir sur l'affirmation indiquée au début de cet article, à savoir que l'une des fonctions de la honte, c'est de permettre au sujet de s'éprouver comme tel. Dans cette approche du champ de la honte, vont être développés à présent les trois temps subjectifs aptes à conceptualiser le traitement psychothérapeutique de la honte:

a) Dans un premier temps, "la honte de la honte" ne permet pas au sujet d'en parler, dans la mesure où c'est la honte elle-même qui interdit sa propre expression: c'est le temps de l'inhibition.

b) Dans un deuxième temps, après avoir apprivoisé la honte dans un cadre approprié à l'accueillir, la mise en mots de la honte par le sujet sera effective: c'est le temps du regard.

4. Le régime alimentaire draconien entrepris par la patiente à cette même période, avait pour motif le désir explicite "de retrouver une image physique positive". 


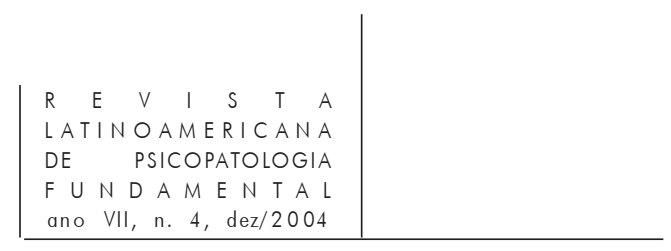

c) L'expression et la reconnaissance de la honte par le sujet vont lui permettre l'accès à une expérience subjective: c'est le temps de l'identité.

Le premier temps: l'inhibition

D'un point de vue métapsychologique, l'inhibition correspond à un processus physiologique actif et réversible, de suspension temporaire d'une fonction par une autre. Le mouvement rétrograde imprimé au sujet par l'importance de l'inhibition s'étend à la sphère somatique et psychique. Les perturbations fonctionnelles se définissent de façon négative par le fait qu'une activité ne peut avoir lieu: "Ces perturbations fonctionnelles, expression d'une limitation du moi, constituent ce que l'on appelle des 'inhibitions"' (Chemama, 1993, p. 147). Le Moi renonce à certaines fonctions pour ne pas avoir à entreprendre un nouveau refoulement, pour ne pas entrer en conflit avec le ça. Mais, écrit Freud, "Relié intimement au ça lui-même, il ne peut écarter défensivement le danger de pulsion qu'en restreignant sa propre organisation et en s'accommodant de la formation de symptôme comme substitut du préjudice porté par lui à la pulsion" (Freud, 1926, p. 68). Le Moi, lorsqu'il est confronté à une épreuve psychique difficile, ici une énorme répression d'affect, connaît un appauvrissement en énergie qui manifeste la restriction fonctionnelle moïque et que caractérise l'expression de l'inhibition.

L'inhibition comme manifestation symptomatique de la honte était manifeste dès le premier entretien. Cette inhibition était à la mesure de la forte mobilisation défensive sous-jacente. D'emblée, Mme M. évoque l'inceste familial mais cette mise en récit factuelle est dénuée d'affects, comme si elle n'avait pas vécu ces violences qu'elle décrit. Dans la dynamique intersubjective, malgré les efforts de restitution d'un tissu associatif, la pensée s'enlisait. Les propos de la patiente portaient sur des évènements traumatiques de sa vie mais ne semblaient réveiller ni association ou réaction affective, l'inhibition venant réprimer toute expression d'affects. Si le clinicien peut entendre la parole du sujet, il pourra l'aider à accéder à une subjectivation que si ce dernier est d'abord en mesure de s'approprier ce qu'il dit. Mme M. illustrait tout à fait cette quête et sa mise en échec. Dans le transfert, elle occupera de nombreuses séances à relater et décrire précisément le contenu et la nature des violences incestueuses subies. À la tentative d'appropriation des faits par la patiente se manifestait la tentative conjointe du clinicien de mettre en représentation ce qui était dit. Mais dans le contre-transfert, je me heurtais régulièrement à l'impossibilité de me représenter la violence et la cruauté des actes subis. C'est comme si, dans la dynamique transféro-contretransférentielle, nous étions prises dans le même gel des mouvements associatifs; 


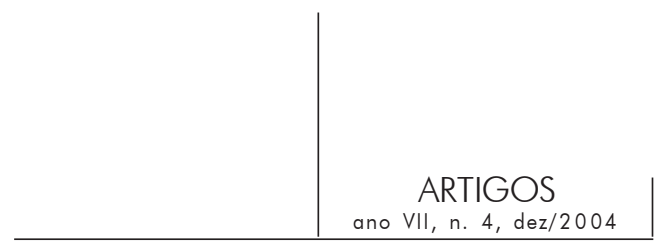

à sa description itérative des faits se conjuguait mon incapacité à les penser, c'està-dire, comme le souligne très justement B. Jacobi, "cette patiente restait dans l'événementiel: elle ne passait pas de l'événement à l'essentiel". ${ }^{5}$ Dans la répétition et la déchéance ressentie, la déliaison pulsionnelle sourdait silencieusement et poursuivait ses desseins destructeurs. Honte invétérée et innocence souillée s'affirmaient dans cette conviction dramatique: "J'ai été humiliée dans mon corps de petite fille et d'adolescente. Cette honte et cette salissure sont indélébiles. Ma vie est gâchée”.

L'abord de l'événement traumatique surviendra dans l'après-coup, fragment par fragment, dans la confusion et le tremblement des mots; ce qui est vecteur de terreur, c'est de formuler l'énoncé et de l'entendre: 'L'effet d'une parole ne tient pas à son expulsion, mais à sa capacité d'inscription pour celui qui peut s'entendre la dire" (Jacobi, 2001, p. 153). Comment faire partager cet indicible, cet impensable de la souffrance au cœur même de la subjectivité? La situation traumatique est paradoxale puisqu'elle génère une souffrance à la fois impensable, non " historicisée" et en même temps incontournable. Ferenczi (1923), qui a joué un rôle essentiel dans la mise en place de repères analytiques propres à permettre de comprendre la notion de traumatisme, explique que suite “... à la découverte et la reconstruction du trauma supposé, suit une série quasi infinie de répétitions dans les séances d'analyse, avec toutes les explosions d'affects inimaginables" (p. 162) comme des réactions de colère, de révolte, d'effroi, de terreur, d'angoisse, de vengeance. Or, et c'est là un point essentiel, l'inceste - qui prend une place très particulière du fait du lien intrinsèque de filiation réel, imaginaire et/ou symbolique - imprime chez la victime des sentiments de culpabilité et de honte, culpabilité de ne pas "avoir pu empêcher cela”, honte "d'avoir fait cela" comme le confiera Mme M. Précipitée dans l'horreur d'une épreuve innommable, la patiente se sent coupable et honteuse de ce dont elle est victime. Culpabilité d'avoir commis une faute, d'avoir transgressé, manquement dans le fait de ne pas avoir pu empêcher cet inceste. Honte de la souillure sexuelle, de la pénétration forcée, honte d'avoir été l'objet de la jouissance de l'autre. Comme l'écrit B. Jacobi (2001), le sujet victime de violences sexuelles éprouve la “... honte d'être devenu déchet, honte d'être déchet par la déchéance infligée” (p. 149). En tant qu'enfant violenté, honte d'avoir été le témoin forcé de cette jouissance prise par l'autre, son père en l'occurrence, dans une proximité sexuelle et corporelle insoutenables et subies.

5. Professeur B. Jacobi, entretien du Mercredi 16 mai 2001. 


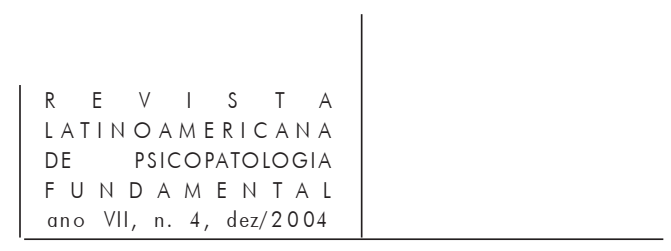

Le deuxième temps: la mise en mots de l'événement traumatique

C'est à partir de la nomination de l'idée de plaisir sous une forme négative que la patiente pourra aborder plus précisément les souvenirs des expériences sexuelles traumatiques, la dénégation du plaisir ressenti revêtant précisément l'indication inverse, à savoir celle justement du plaisir pris. Ainsi dans l'expression "N'allez pas penser que j'ai eu du plaisir" la patiente laisse-t-elle parler le sujet de l'inconscient, sous une forme niée. Ce qui nous amène à considérer que $l a$ dynamique d'apparition de la honte surgirait dans cet entre-deux, entre échec et réussite, dans cet intervalle d'abord, entre refoulement et dénégation. L'échec du refoulement se manifeste par la honte qui exprime l'auto-dénonciation de l'individu qui a dérogé à son Idéal du Moi et son Moi-Idéal: la déception d'une vie ordinaire, stable, normale peu conforme à l'Idéal du Moi du sujet et d'autre part la déchéance d'un Moi-Idéal relégué aux limbes de la nullité absolue, de la non valeur si ce n'est sous forme d'objet sexuel des fantasmes de son père mais aussi de déchet à évacuer, ce dernier la traitant, selon ses dires, comme "une merde".

Ainsi, dans la dynamique transférentielle, Mme M. me prêtait une pensée qui n'était autre que la sienne propre, affirmée sur le mode d'une négation: "J'ai peur que vous pensiez que j'avais du plaisir dans ses relations incestueuses". Dans cette honte du plaisir éprouvé, c'est d'abord une honte qui protège de l'effroi, celle du plaisir pris. Lorsque plus tard, la patiente pourra remplacer la mention "Je n' ai pas eu de plaisir dans ces actes" par la révélation "J'ai honte de ces actes et de ce que j'ai fait" s'ouvrent les prémices de la reconnaissance du sens que ces pensées impliquaient. Autrement dit, le dépassement de "la honte de la honte" permet de nommer la barbarie et d'ouvrir la voie psychique à une ébauche d'élaboration. La patiente va déployer, dans la pénombre des mots et des silences, les bribes des scènes incestueuses, les violences physiques, sexuelles, dans une tentative de liaison des impressions sensorielles, émotions et représentations. L'accès à une expérience subjective commence ici, dans la réintroduction du traumatisme dans la temporalité de la thérapie, dans sa restitution au sein de l'histoire individuelle et de son économie psychique.

Cette évocation du traumatisme qui s'effectue dans la honte n'est pas sans rappeler la formule de C. Barazer (2000), qui situe la honte “ $\ldots$ au carrefour de ce triple processus d'exposition, de dépossession et de déchéance de soi face à l'autre" (p. 9). Dans ce sens, l'exposition de soi concerne le sujet ayant été crûment l'objet d'attaques portant sur son intégrité physique, somatique et sexuelle, la dépossession de soi concerne essentiellement le corps propre, brutalement confronté à l'irruption et la violence pulsionnelles de l'agresseur qui s'approprie le corps de sa victime comme objet de jouissance; de cette précipitation dans l'horreur traumatique, la déchéance ressentie en porte le cruel 


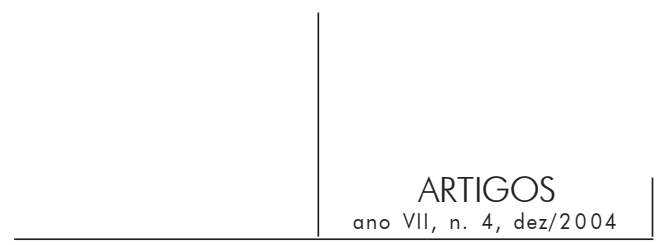

témoignage: infigurable de la douleur, présence inoubliable et impartageable de la scène traumatique lorsque le sujet est brutalement ravalé au rang de chose, d'objet sexuel voire de déchet abject. Ce tryptique d'exposition, de dépossession et de déchéance de soi rejette le sujet dans une incommensurable solitude, dans une unicité honteuse, indécente, dépouillée de toute capacité de subjectivation et de symbolisation.

Le troisième temps: le processus de (ré)appropriation subjective de l'événement traumatique par le sujet

À partir de cette vignette clinique, nous voyons comment, dans le cadre d'entretiens psychothérapiques, la reconnaissance de l'éprouvé de la honte par Madame M. a fonctionné comme (ré)amorçage d'un lien dans une situation d'isolement et de souffrance. Se reconnaître et se faire reconnaître sujet honteux, c'est déjà s'affirmer sujet. Comme l'écrit C. Miollan (1998), “... pouvoir montrer sa honte, c'est obtenir un regard de l'autre qui servira de contenant provisoire" (p. 164). Ainsi, cette demande de (ré)instauration de liens se nourrit et se maintient dans l'échange des regards, regard ayant à la fois une fonction de contenant et une dimension sociale.

Lorsque l'inscription pathologique de la honte est venue figer le sujet dans sa sphère psychique, et l'exclure de l'espace transitionnel et fondamental de la parole, il y a nécessité que s'énonce cette honte et ces béances, afin que soit recueilli, (ré)approprié et transmis ce qui jusque-là n'avait pu se dire. En ce sens, lorsque la patiente met en discours sa honte, elle devient, dès lors, un objet de discours, un concept mentalisé, ce qui va permettre au sujet de prendre de la distance. Lorsqu'elle dit “j'ai honte”, elle a déjà conscience de sa honte et elle change le statut de la honte qui, d'attribut, devient objet. Du coup, la problématique de l'adjectivation et de la subjectivation de la honte s'inscrit dans une temporalité de la thérapie. Tant que cette honte n'est pas dite, elle envahit le sujet et "colle" littéralement à son moi. L'accès à une expérience subjective commence ici, dans la réintroduction du traumatisme dans la temporalité de la thérapie, dans sa restitution au sein de l'histoire individuelle et de son économie psychique. Ainsi, d'une parole de honte sans adresse ou en souffrance d'adresse s'est déployée la verbalisation de la honte, réinscrivant dans l'actuel la figure parfois pétrifiée, en tous les cas figée, de cette épreuve. Enfin, l'analyse de ce fragment clinique montre que le traitement psychothérapeutique de la honte, dans la succession des différentes temporalités psychiques et de leur étroite combinaison, passe par les trois temps suivants: l'inhibition, la mise en mots de la honte, l'affirmation et la reconstruction de l'identité. 


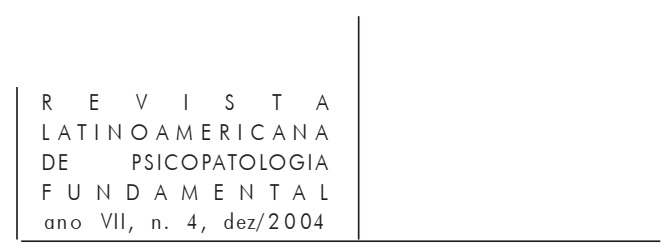

\section{Conclusion}

Notre propos a insisté, à travers une évocation clinique, sur cette cooccurrence entre traumatisme sexuel et sentiment de honte. Plus globalement, nous avancerons que cette articulation, qui trouve des fondements théoriques et cliniques, est renforcée par notre pratique de clinique analytique qui questionne régulièrement l'expression de sentiments de honte chez des sujets victimes de traumatismes sexuels, entravant considérablement et durablement leur vie psychique. La honte a une valeur structurante dans le maintien et la reconstruction subjective du sujet: elle témoigne que même réduit à être l'instrument de jouissance de l'autre dans une violence sans nom, le sujet n'est pas totalement assujetti à cet autre, agresseur, violeur, bourreau. Par la honte, le sujet témoigne donc qu'il continue à s'éprouver comme tel.

En conclusion, la honte et son expression scandent une problématique de subjectivation, elles indiquent et amènent une réinscription dans la temporalité. Elles permettent un changement de registre entre l'identification spéculaire et l'identification symbolique, et une capacité nouvelle à s'adresser à l'autre, le petit autre et le grand Autre.

\section{Références}

ANDrÉ, S. L'imposture perverse. Paris: Seuil, 1993.

Barazer, C. Hontes sans issue. Documents \& Débats, Bulletin Intérieur de l'Association Psychanalytique de France, n. 52, 2000.

Blanc, E. Thèse pour le Doctorat en Sciences Humaines: L'inceste: la prohibition et l'inter/dit. Université de Nice Sophia-Antipolis, Nice, 1997.

Chemama, R. (1993). Dictionnaire de la psychanalyse. Paris: Larousse, 1995.

Clavreul, J. Le désir et la loi. Paris: Denoël, 1987.

Ferenczi, S. (1923). Journal clinique. Paris: Payot, 1985.

Freud, S. (1896). Nouvelles remarques sur les psychonévroses de défense. In: Névrose, psychose et perversion. Paris: P.U.F., 1973.

(1905). Trois essais sur la théorie sexuelle. Paris: Gallimard, 1987.

(1912). Totem et tabou. Paris: Payot, 1965.

(1915). L'inconscient. In: Métapsychologie. Paris: Gallimard, 1968.

(1921). Psychologie des foules et analyse du moi. In: Essais de psychanalyse. Paris: Payot, 1981. 


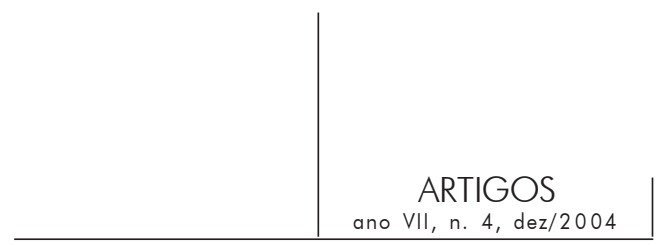

(1923). Le moi et le ça. In: Essais de psychanalyse. Paris: Payot, 1981.

(1926). Inhibition, symptôme et angoisse. Paris: Quadrige/P.U.F., 1993.

JАCOBI, B. (1998). Les mots et la plainte. Toulouse: Erès.

Introduction sur un malaise supposé. Colloque du CIRPC/Université de Provence d'Aix-Marseille I et Université Louis Pasteur de Strasbourg - "Malaise dans la filiation”. Aix-en-Provence, 27 e 28 novembre 1999. Communication orale.

De la honte à la plainte. In: Victime-Agresseur Tome 1- Le traumatisme sexuel et ses devenirs. Lecques: Les Editions du Champ Social, 2001. p. 147-5.

LaCAN, J. (1945). Le stade du miroir comme formateur de la fonction du Je. In: Écrits. Paris: Seuil, 1966. p. 93-100.

(1948). L'agressivité en psychanalyse. In: Écrits. Paris: Seuil, 1966. p. 101-24.

(1960). Subversion du sujet et dialectique du désir dans l'inconscient freudien. In: Écrits. Paris: Seuil, 1966. p. 793-827.

Miollan, C. Inceste, une écoute post-traumatique. Cliniques Méditerranéennes: Exil et migrations dans la langue. Toulouse: Erès, n. 55-6, 1998.

SCHNEIDER, M. Le franchissement du seuil, Freud et la thématique du regard. Cliniques Méditerranéennes: Césure du regard, Cliniques du visuel. Toulouse: Erès, n. 51-2, 1996.

\section{Resumos}

O tratamento psicoterapêutico das crianças ou dos adultos vítimas de traumatismos sexuais revela um sentimento de vergonha característico. A primeira hipótese aqui considerada toma em conta as duas instâncias psíquicas da personalidade que são o Ideal do Eu e o Eu Ideal, cujos elementos determinantes são: por um lado, a relação do Ideal do Eu com a experiência de uma agressão sexual e da ferida narcísica irreversível que ela provoca, e por outro lado, o Eu Ideal em relação ao sentimento de degradação implicado na vergonha decorrente do traumatismo sexual, vergonha de ser só a coisa, o objeto, o dejeto do agressor. A segunda hipótese postula que no dispositivo psicoterapêutico a expressão e o reconhecimento pelo sujeito da vergonha como pertence subjetivo demonstram que não há perversão $e$ constituem um ponto de apoio essencial na afirmação e na reconstrução da identidade. Nossa reflexão insiste, através de uma evocação clínica, nessa co-ocorrência de traumatismo sexual e sentimento de vergonha.

Palavras-chave: Identidade, narcisismo, psicoterapia, sentimento de vergonha, trauma sexual 


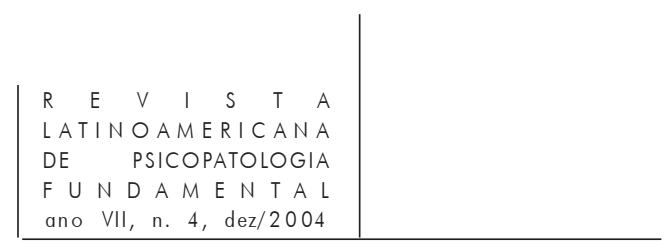

El tratamiento psicoterápico de niños o adultos víctimas de traumatismos sexuales hace aparecer un sentimiento de vergüenza característico. La primera hipótesis aquí levantada lleva en cuenta dos instancias psíquicas de la personalidad como son el Ideal del Yo y el Yo ideal, cuyos elementos determinantes son: por un lado, la relación del Ideal del Yo con la experiencia de atentado sexual y la herida narcisista que provoca, por otro lado, el Yo Ideal en relación con la degradación experimentada y la vergüenza consecutiva al traumatismo sexual, vergüenza de no ser mas que la cosa, el objeto, el deyecto del agresor. La segunda hipótesis postula que en el dispositivo psicoterapéutico, la expresión y el reconocimiento de la vergüenza como algo que pertenece al sujeto señala que no ha habido perversión y constituye un punto de apoyo esencial en la afirmación y la reconstrucción de la identidad. Nuestra reflexión insiste, a través de una evocación clínica, en la concomitancia entre traumatismo sexual y vergüenza.

Palabras claves: Identidad, narcisismo, psicoterapia, sentimiento de vergüenza, trauma sexual

The psychotherapeutic treatment of child and adult victims of sexual trauma reveals a characteristic feeling of shame. The first hypothesis envisaged considers two psychological aspects of the personality: Ideal Ego and Ego Ideal. The critical elements for these are, on the one hand, the reaction of the Ideal Ego to the experience of a sexual attack and the irreversible narcissistic damage it causes. On the other hand, the Ego Ideal suffers from the abandonment felt due to the process of shame following a sexual trauma, the shame of seeing oneself as a mere object, something used and discarded by the aggressor. In this article we discuss the second hypothesis, to the effect that in psychotherapeutic treatment, the subject's expression and recognition of shame constitute an essential aid for affirming and reconstructing identity. Through a clinical analysis, our reflection focuses on the occurrence of both sexual trauma and the feeling of shame.

Key words: Feeling of shame, identity, narcissism, psychotherapy, sexual trauma

Versão inicial recebida em setembro de 2003

Versão revisada recebida em novembro de 2004 